\title{
Asymptotic analysis of first passage time in complex networks
}

\author{
Hon WAi LAU ${ }^{(a)}$ and K. Y. SzEto ${ }^{(b)}$ \\ Department of Physics, The Hong Kong University of Science and Technology, Hong Kong, China
}

PACS 05.40.Fb - Random walks and Levy flights

PACS 89.75. Hc - Networks and genealogical trees

PACS 87.10.-e - General theory and mathematical aspects

\begin{abstract}
The first passage time (FPT) distribution for random walk in complex networks is calculated through an asymptotic analysis. For network with size $N$ and short relaxation time $\tau \ll N$, the computed mean first passage time (MFPT), which is inverse of the decay rate of FPT distribution, is inversely proportional to the degree of the destination. These results are verified numerically for the paradigmatic networks with excellent agreement. We show that the range of validity of the analytical results covers networks that have short relaxation time and high mean degree, which turn out to be valid to many real networks.
\end{abstract}

Network is important in many areas of science and engineering as a mathematical representation of the interaction of complex real systems [1,2] such as the Internet, social network, etc. Some of the common topological properties for many real world networks, such as small-world and scale free properties [1], and fractal scaling [3] have been investigated. Recent research effort has begun to address the dynamical properties and critical phenomenon in complex network. Random walk problem, due to its simplicity, is therefore a key to the understanding of the propagation of dynamical quantities in network. The propagation of a random walker, which can represent the spreading of some signal in specific application, can be measured by the time needed to reach a selected node. In this regard, the first passage time (FPT) that measures the first time the random walker visiting a given destination, is critical to many events triggered by the walker, such as epidemics spreading in social network [5], neuron firing dynamics [6] and various target search process [7,8]. Instead of the whole distribution, the mean first passage time (MFPT) is widely studied in different lattices with unbounded domain 8], and bounded domain with arbitrary shape of boundary [9. Although the general solution of MFPT has been found [10], the explicit relation between first passage time and structural properties of network is still unknown. A recent work has related MFPT to the distance between source and destination 11] for fractal networks, which use the analysis of pseudo Green function for finite

\footnotetext{
(a) E-mail: lau65536@gmail.com

(b) E-mail: phszeto@ust.hk
}

lattice [9]. Nonetheless, it has been argued that this result should only be applicable to the deterministic fractal network with homogeneous degree distribution [12]. In comparison with most deterministic fractal with fixed degree, real world networks typically have strong inhomogeneity in degree distribution. Thus, it suggests that the degree of nodes should play an essential role for the random walk in complex network and a treatment differently from the analysis of fractal network should be used.

For many real networks, the number of nodes in a given data set is usually not big, so that a rigorous analysis on its properties is usually difficult. Hence, schemes to adjust the size of network have been proposed [3]13], but it is difficult to preserve structural properties without knowing the underlying construction mechanism. Computing the MFPT for networks with a particular property can therefore be useful for real world network. Our method should be complementary to the pseudo Green function approach 11.

In this paper, we will provide an analytical expression for the asymptotic first passage time distribution function, from which all moments, including the mean, can be computed and compared with numerical results for real and paradigmatic networks. We focus on the study of random walk in complex networks with short relaxation time irrespective of the type of network. In contrast to the pseudo Green function approach, our asymptotic analysis can also reveal the local dynamical properties around the destination. In particular, it allows us to compute a good approximation of the decay rate of FPT distribution and 


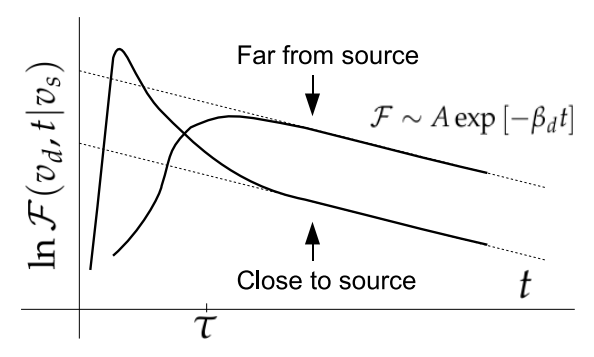

Figure 1: Typical FPT distribution for a destination $v_{d}$. At the long time scale $t \gg \tau$, FPT distribution decays exponentially. For the destination close to the source, the walker can reach the destination in a short time, resulting in a peak higher than the asymptotic curve. If the destination is far from the source, the walker has to visit many other nodes before the destination, resulting in a slow increase of FPT distribution.

MFPT for networks with short relaxation time. We further argue that these expressions are approximate bounds for other general complex networks. Since most real world networks have short relaxation time, we find that our analytical calculation produces excellent comparison to numerical results on the MFPT for paradigmatic as well as real world networks.

Formulation. - We consider a finite undirected network which consists of a set of nodes $\left\{v_{1}, v_{2}, \ldots, v_{N}\right\}$ that are connected by edges. The set of neighbors of a node $v$ is denoted by $\operatorname{Nei}(v)$. Let $\mathcal{P}(v, t)$ be the probability of a random walker located at node $v$, at time step $t$. At each time step, the walker can move from the current node $v_{i}$ to one of its nearest neighbor node $v_{j} \in \operatorname{Nei}\left(v_{i}\right)$, with equal probability $1 / k_{i}$. Hence, the master equation of random walk is

$$
\mathcal{P}\left(v_{i}, t+1\right)=\sum_{v_{j} \in \operatorname{Nei}\left(v_{i}\right)} \frac{\mathcal{P}\left(v_{j}, t\right)}{k_{j}}
$$

For the random walk problem in a finite domain, there is a characteristic relaxation time $\tau$ such that equilibrium is essentially reached when $t \gg \tau$. We denote the equilibrium probability distribution by $\mathcal{P}^{\infty}(v)$ for $t \rightarrow \infty$. By the principle of detailed balance, the net flow of walker probability $\mathcal{P}^{\infty}\left(v_{i}\right) / k_{i}-\mathcal{P}^{\infty}\left(v_{j}\right) / k_{j}$ is zero along each edge at equilibrium. Hence, the equilibrium probability is proportional to the node degree $\mathcal{P}^{\infty}(v) \propto k_{v}$. After normalization, we get the Kac's result [9], $\mathcal{P}^{\infty}(v)=k_{v} / N\langle k\rangle$, where $\langle k\rangle$ is the mean degree of network. Therefore, at equilibrium, the walker probability moving along each edge, in both directions, is equal to $1 / N\langle k\rangle$.

Let $\mathcal{F}\left(v_{d}, t \mid v_{s}\right)$ be the FPT distribution from a source node $v_{s}$ to a destination node $v_{d}$ that takes time $t$. By adding a sink at the destination, the FPT distribution can be found by computing the probability of the walker trapped at the sink. For a network with sink, we denote the probability that the walker is located at node $v$, at time $t$ by $\mathcal{W}(v, t)$. Note that $\mathcal{W}(v, t)$ is the analog of $\mathcal{P}(v, t)$, which addresses a network without sink. The FPT distribution for a sink at $v_{d}$ can be computed by the following equations:

$$
\begin{cases}\mathcal{W}(v, 0)=\delta_{v v_{s}} & \forall t>0 \\ \mathcal{W}\left(v_{d}, t\right)=0 & \forall v \in V-\left\{v_{d}\right\} \\ \mathcal{W}(v, t)=\sum_{u \in \operatorname{Nei}(v)} \frac{\mathcal{W}(u, t-1)}{k_{u}} & \\ \mathcal{F}\left(v_{d}, t \mid v_{s}\right)=\sum_{u \in \operatorname{Nei}\left(v_{d}\right)} \frac{\mathcal{W}(u, t-1)}{k_{u}} & \end{cases}
$$

The first equation is the initial condition. The second equation is the absorbing boundary condition at the destination. The third equation is the random walk transition probability corresponding to (1). These three equations define the random walk problem with a sink located at $v_{d}$. The last equation introduces the method to find the FPT distribution, which is given by the incoming probability flux flowing towards the destination. Moreover, removal of the walker at the sink decreases the total walker probability, or the survival probability $\mathcal{W}_{\text {total }}(t)$ defined by $\mathcal{W}_{\text {total }}(t)=\sum_{v \in V} \mathcal{W}(v, t)$. Hence, the FPT distribution and the total walker probability are related by

$$
\mathcal{F}\left(v_{d}, t \mid v_{s}\right)=\mathcal{W}_{\text {total }}(t-1)-\mathcal{W}_{\text {total }}(t)
$$

as the random walker is absorbed by the destination node at time $t$.

Now, we perform asymptotic analysis of FPT distribution. When $t \gg \tau$, the initial information of the source node is washed away, so walker probability decreases uniformly for each node in the network. In this case, $\mathcal{W}(v, t)$ can be separated into two parts:

$$
\mathcal{W}(v, t) \sim \mathcal{W}(v) \mathcal{W}_{\text {total }}(t), \quad t \gg \tau
$$

where $\mathcal{W}(v)$ is a time independent probability distribution that depends on network topology and the location of destination. By substituting Eq. (4) back into the last equation in 22, we can get a structural factor $\beta_{d}$ that depends on the destination node $v_{d}$ :

$$
\beta_{d}=\sum_{u \in \operatorname{Nei}\left(v_{d}\right)} \frac{\mathcal{W}(u)}{k_{u}}
$$

and the asymptotic form of FPT distribution $\mathcal{F}\left(v_{d}, t \mid v_{s}\right) \sim$ $\beta_{d} \mathcal{W}_{\text {total }}(t-1)$ for $t \gg \tau$. Solving this equation along with Eq. (3), we have $\mathcal{W}_{\text {total }}(t) \sim\left(1-\beta_{d}\right) \mathcal{W}_{\text {total }}(t-1)$ and so $\mathcal{W}_{\text {total }}(t) \propto\left(1-\beta_{d}\right)^{t}$ asymptotically. As we will show later, $\beta_{d}$ is small, so that $\mathcal{W}_{\text {total }}(t) \propto \exp \left[-\beta_{d} t\right]$. With the known asymptotic form of $\mathcal{W}_{\text {total }}(t)$, we can conclude that the FPT distribution is $\mathcal{F}\left(v_{d}, t \mid v_{s}\right) \sim A \exp \left[-\beta_{d} t\right]$. Thus, FPT distribution has an exponential tail with decay rate $\beta_{d}$ as shown in Fig. 1. In addition, we now know a method to compute decay rate analytically by Eq. (5), provided that $\mathcal{W}(u)$ of all neighbors of destination $v_{d}$ is known.

Decay rate of FPT distribution. - After deriving the exponential tail of the FPT distribution, our next task is to compute the decay rate $\beta_{d}$ for the network with short relaxation time $\tau$ such that $\tau \ll 1 / \beta_{d}$. Physically, $\tau$ represents the time to reach equilibrium in a network 

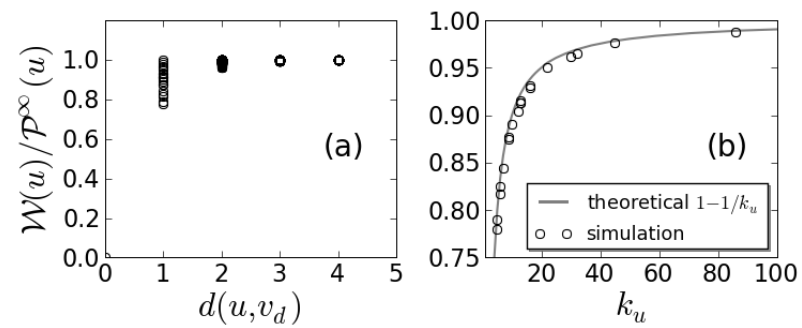

Figure 2: For $t \gg \tau$, we show the deviation $\mathcal{W}(u) / \mathcal{P}^{\infty}(u)$ of the time independent probability distribution $\mathcal{W}(u)$ from the equilibrium distribution $\mathcal{P}^{\infty}(u)$ when there is no sink, as a function of (a) distance from destination $v_{d}$, (b) degree of nearest neighbors $u \in \operatorname{Nei}\left(v_{d}\right)$ which correspond to the distance $=1$ in (a). The network used is the BA model with $N=10000$ and $\langle k\rangle=10$.

without sink, and $1 / \beta_{d}$ is the approximate time scale for the decrease in total walker probability of the network when there is a sink to absorb the random walker. For network with short relaxation time, the removal of walker probability around the destination can be accomplished rather quickly. On the other hand, the total probability decreases with a much slower rate and this result in a quasi-equilibrium state. This physical picture implies that the quantity $\mathcal{W}(u)=\lim _{t \gg \tau} \mathcal{W}(u, t) / \mathcal{W}_{\text {total }}(t)$ is approximately equal to $\mathcal{P}^{\infty}(u)$. This can be observed for the Erdos-Renyi (ER) network and Barabasi-Albert (BA) network which have very short relaxation time (see Table 1). One of them is illustrated numerically in Fig. 2 a. In the simplest case, it is reasonable to assume that $\mathcal{W}(u)=\mathcal{P}^{\infty}(u)$ for $d\left(u, v_{d}\right) \geq 1$. While this assumption is substituted back to Eq. (5), the decay rate is $\beta_{d}=k_{d} / N\langle k\rangle$. It scales linear with the degree of destination and scales inversely with the size of the network. Thus, for a large network, $\beta_{d}$ can be very small.

Next, we proceed to a better approximation for the dependence of $\mathcal{W}(u)$ on the $k_{u}$ around the destination. For a nearest neighbor $u$ of the destination, $\mathcal{W}(u)$ has to take a smaller value than $\mathcal{P}^{\infty}(u)$ to compensate the flow of walker toward the sink. Hence, it is natural to expect that $\mathcal{W}(u) \leq \mathcal{P}^{\infty}(u)$. Rather than assuming $\mathcal{W}(u)=\mathcal{P}^{\infty}(u)$ for nodes other than destination, a refined assumption can be made for the next nearest neighbors:

$$
\mathcal{W}(u, t)=\mathcal{P}^{\infty}(u) \mathcal{W}_{\text {total }}(t), \quad d\left(u, v_{d}\right) \geq 2
$$

With this assumption, $\mathcal{W}(u)$ for $u \in \operatorname{Nei}\left(v_{d}\right)$ has to be found before computing the decay rate. Here, nodes with distance two from the destination are treated as the reservoir of walker probability. We thus assume that each edge of node $v$, that are of distance greater than 1 away from the destination, $d\left(v, v_{d}\right) \geq 2$, has probability $1 / N\langle k\rangle$ moving out at each time step.

Now, let us focus on a nearest neighbor node $u$ of the destination. Since there is no probability flowing from $v_{d}$ to $u$, therefore, only $k_{u}-1$ neighbors of $u$ have probability
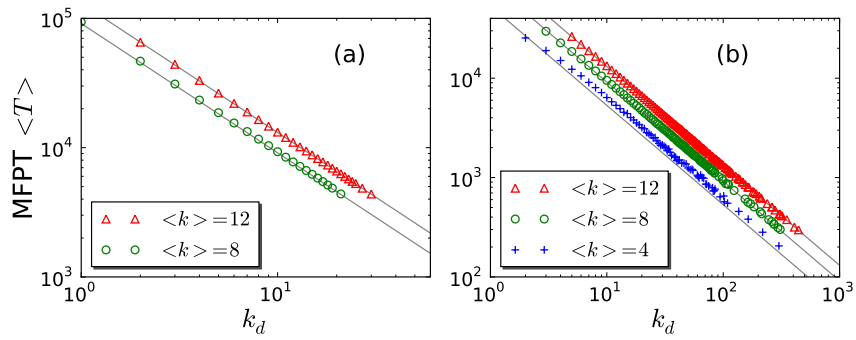

Figure 3: (color online) MFPT vs degree of destination $k_{d}$ for the (a) ER and (b) BA model, with $N=10000$. The points are simulation result and the lines are the theoretical prediction.

flowing into $u$. If there are no edges connecting between neighbors of $v_{d}$, i.e., assuming zero clustering coefficient of $v_{d}$, then each edge contributes $1 / N\langle k\rangle$ to $u$ from the second neighbor of $v_{d}$, or $\mathcal{W}(u)=\left(k_{u}-1\right) / N\langle k\rangle$. If there are some edges connecting neighbors of $v_{d}$, i.e., for the case of non-zero clustering coefficient, then some neighbors of $u$ are also neighbors of $v_{d}$. In this case, nodes in $\operatorname{Nei}\left(v_{d}\right)$ have value less than $\mathcal{P}^{\infty}$ and the probability moving out has value less than or equal to $1 / N\langle k\rangle$. The exact value can be found by solving $\mathcal{W}(u)$ for all $u \in \operatorname{Nei}\left(v_{d}\right)$ simultaneously and it is upper bounded by

$$
\mathcal{W}(u) \leq\left(\frac{k_{u}-1}{k_{u}}\right) \mathcal{P}^{\infty}(u), \quad d\left(u, v_{d}\right)=1
$$

As shown in Fig. 2b for the BA model, this result fits well with the simulation of $\mathcal{W}(u)$ and the equality sign holds approximately. In general, this upper bound can be reached for networks with very short relaxation time. Now we put Eq. (7) into Eq. (5) to get

$$
\beta_{d} \leq \frac{k_{d}}{N\langle k\rangle}\left(1-\frac{1}{k_{d}} \sum_{u \in \operatorname{Nei}\left(v_{d}\right)} \frac{1}{k_{u}}\right)
$$

where the last term is the mean of inverse degree of neighbor that can be approximated by mean field. Let's recall that the probability of selecting one of nearest neighbors with degree $k$ for uncorrelated network is $P(k) k /\langle k\rangle$ [14, where $P(k)$ is the degree distribution of the network. Similar to the computation of mean degree of neighbor in Ref. [15, the mean of inverse degree of neighbor is $\int_{0}^{\infty}(P(k) k /\langle k\rangle) \frac{1}{k} d k=\frac{1}{\langle k\rangle}$, so

$$
\beta_{d} \leq \frac{k_{d}}{N\langle k\rangle}\left(1-\frac{1}{\langle k\rangle}\right)
$$

which suggests that the actual decay rate obtained in simulation is slower than this upper bound. Note that this result is consistent with our basic hypothesis $\tau \ll 1 / \beta_{d}$ if $\tau \ll N$. We find that this theoretical decay rate is in excellent agreement with the simulation result for the ER and BA network (see Table 1). 
Mean first passage time. - Since FPT distribution is normalized for a finite undirected network, $\int_{0}^{\infty} \mathcal{F}\left(v_{d}, t \mid v_{s}\right) d t=1$, it can be split asymptotically as the sum of short time scale and long time scale as:

$$
\int_{0}^{c} \mathcal{F}\left(v_{d}, t \mid v_{s}\right) d t+\int_{c}^{\infty} A e^{-\beta_{d} t} d t \sim 1, \quad c \gg \tau
$$

where $c$ is a cutoff value. In a finite domain, FPT distribution always rise from 0 at $t=0$ and decreases with exponential tail [8] when $t \rightarrow \infty$ (see Fig. 11). For a destination node far from the source node, $\mathcal{F}\left(v_{d}, t \mid v_{s}\right)$ is bounded above by $A e^{-\beta_{d} t}$ within $0<t<c$. Hence, for short relaxation time $\tau \ll c \ll 1 / \beta_{d}$, the first term is bounded by $\int_{0}^{c} A e^{-\beta_{d} t} d t \sim 0$ which contributes negligibly small value to the sum. By extending the domain from $c$ to 0 for the dominant second term, we obtain $A \sim \beta_{d}$ by computing $\int_{0}^{\infty} A e^{-\beta_{d} t} d t \sim 1$. Similar analysis can be performed for MFPT $\langle T\rangle=\int_{0}^{\infty} t \mathcal{F}\left(v_{d}, t \mid v_{s}\right) d t$ and the result is $\langle T\rangle \sim 1 / \beta_{d}$. Using the decay rate derived in Eq. (9), the MFPT is given by

$$
\left\langle T\left(v_{d}\right)\right\rangle \geq \frac{N\langle k\rangle}{k_{d}}\left(\frac{1}{1-\langle k\rangle^{-1}}\right)
$$

which is a lower bound for the MFPT. We can get a similar approximate result for $\left\langle T\left(k_{d}\right)\right\rangle$ of MFPT averaged over all node pairs with same destination degree because this average is dominated by nodes separated far away. Networks with short relaxation time $\tau \ll N$ such as the ER and BA model have diameter of order $\mathcal{O}(\ln N)$ and the number of nodes grow exponential as the distance increase. So the MFPT is dominated by the nodes with separation of order $\mathcal{O}(\ln N)$. We see excellent agreement of our theory with numerical results for the ER and BA networks in Fig. 3. However, for networks with fractal scaling [3], the diameter scales as $\mathcal{O}\left(N^{\alpha}\right)$ for some $\alpha$, so that more details about the structure are required to compute MFPT. Hence, we do not expect our result is good when applied to those fractal networks. Note that Eq. (11) is compatible with a previous numerical result [16] suggesting that $\left\langle T\left(k_{d}\right)\right\rangle \propto 1 / k_{d}$, in which we have also computed the explicit form and shown that it is the lower bound of MFPT of networks with short relaxation time. With the method we used, higher moments of FPT distribution can also be computed as $\left\langle T^{n}\left(k_{d}\right)\right\rangle \approx n ! \beta_{d}^{-n}$. Note also that our derivation depends on the property of short relaxation time regardless of the size of network. In addition, the mean first passage time $\langle T\rangle_{G}$ of the whole network $G$ can be computed by taking weighted average over the degree distribution as $\langle T\rangle_{G}=\sum P(k)\langle T(k)\rangle$. Since $P(k) \rightarrow 0$ as $k \rightarrow \infty$ and $\langle T(k)\rangle \propto 1 / k$ for the networks considered in this paper, we have $\langle T\rangle_{G} \propto N\langle k\rangle$ which grows linear in the network size.

Real world networks. - We now test the applicability of our theory to real world networks which may have long relaxation time and structural inhomogeneity.
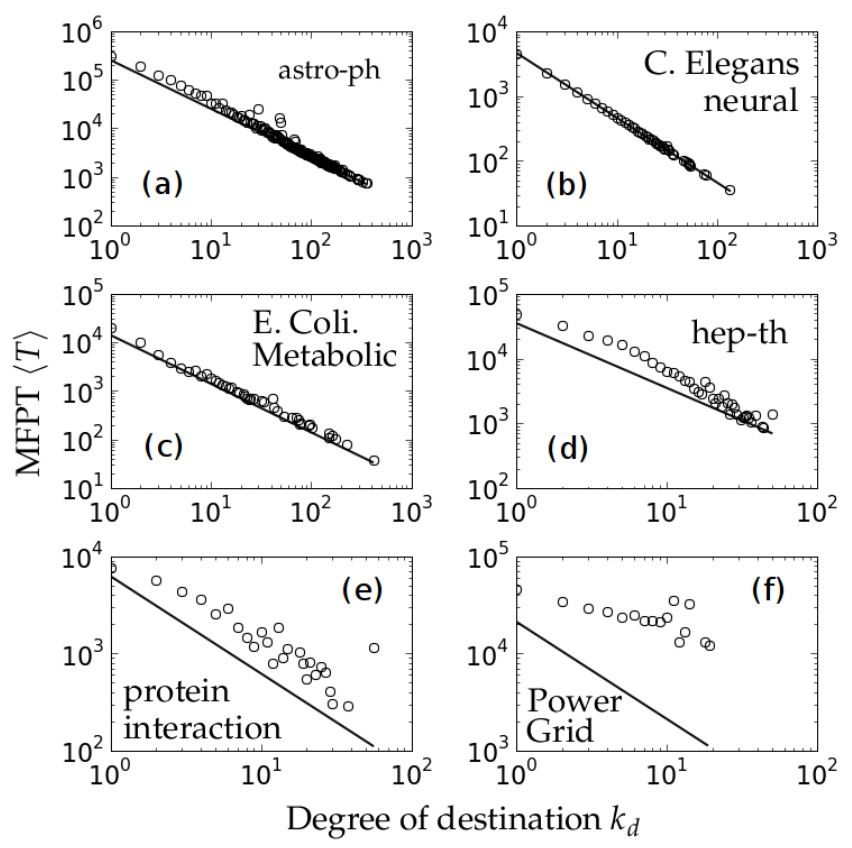

Figure 4: MFPT vs degree of destination $k_{d}$ for real world networks. The circles represent simulation result and the curves represent computed MFPT.

The networks we examined are the Coauthorship network (astro-ph, cond-mat, hep-th) 17], C. Elegans neural network [19], E. Coli. Metabolic network 20], Yeast protein interaction 21] and Western State Power Grid [22]. In the simulation, only the largest connected component of each undirected network is considered.

The random walk properties and MFPT for different networks are presented in Table 1 and Fig. 4. The relaxation time $\tau$ is computed by $\tau=-1 / \ln \left(\left|\lambda_{2}\right|\right)$, where $\lambda_{2}$ is the second largest eigenvalue ${ }^{1}$ of the time evolution operator Eq. (1), or the random walk matrix (see [23]). From Table 1, we can draw the following observations. (1) The decay rates are bounded by our theoretical value for all networks tested $\left(\left\langle\beta_{d}^{*} / \beta_{d}\right\rangle\right.$ is always less than 1$)$. This observation strongly suggests that our result of decay rate can be applied to real world networks. (2) The inverse of the decay rate is approximated equal to the network size for networks with short relaxation time, in agreement with Eq. (9) (3) For BA and ER networks, the relaxation time $\tau$ is short, of the order $\mathcal{O}(\ln N)$. In fact, the relaxation time of a BA network is shorter than the corresponding ER network with the same size and mean degree, which implies that the propagation in the BA network is faster than the ER network. This is caused by the shorter diameter of the BA network $\mathcal{O}(\ln N / \ln \ln N)$, while for ER

\footnotetext{
${ }^{1}$ E. Coli. metabolic network is a bipartite network that has no odd loops, so its second largest eigenvalue is -1 which corresponding to a state oscillating between two different partitions. Nevertheless, the equilibrium for odd and even time series can be reached separately and the first passage time is still well defined. Instead, the third eigenvalue $\lambda_{3}$ can be used to represent the approximate relaxation time to reach the oscillating state.
} 
Table 1: Random walk properties of different paradigmatic and real world networks, with size $N$, mean degree $\langle k\rangle$, relaxation time $\tau$, simulation decay rate $\beta_{d}^{*}$ and theoretical decay rate $\beta_{d}$ given by Eq. (9). The method to compute $\tau$ is described in text and the $\beta_{d}^{*}$ can be found numerically after a long run by using Eq. (5).

\begin{tabular}{|c|c|c|c|c|c|}
\hline Networks & $N$ & $\langle k\rangle$ & $\tau$ & $\frac{1}{\left\langle\beta_{d}^{*}\right\rangle}$ & $\left\langle\frac{\beta_{d}^{*}}{\beta_{d}}\right\rangle$ \\
\hline ER model & 10000 & 12 & 1.7 & 10996 & 0.99 \\
\hline BA model & 10000 & 12 & 1.6 & 10985 & 1.00 \\
\hline BA model & 10000 & 8 & 2.3 & 11624 & 0.99 \\
\hline BA model & 10000 & 4 & 5.6 & 14457 & 0.98 \\
\hline Coauthorship network, astro-ph 17,18 & 14845 & 16.1 & 304 & 18435 & 0.82 \\
\hline C. Elegans neural network 19 & 297 & 14.5 & 4.6 & 323 & 0.99 \\
\hline Coauthorship network, cond-mat 17,18 & 13861 & 6.4 & 139 & 23601 & 0.70 \\
\hline E. Coli. Metabolic network 20 & 2268 & 5.0 & $(16)$ & 3190 & 0.92 \\
\hline \begin{tabular}{ll|} 
Coauthorship network, hep-th & 17 \\
\end{tabular} & 5835 & 4.7 & 179 & 10955 & 0.69 \\
\hline Yeast protein interaction 21 & 1458 & 2.7 & 120 & 3398 & 0.83 \\
\hline Western State Power Grid 22 & 4941 & 2.7 & 3689 & 30008 & 0.33 \\
\hline
\end{tabular}

it is of order $\mathcal{O}(\ln N)$ 24]. Our theory of FPT distribution works very well for the ER and BA networks, as they both have short relaxation times. (4) For those real world networks with relaxation time $\tau$ and the inverse of decay rate $1 /\left\langle\beta_{d}^{*}\right\rangle$ smaller by two orders of magnitude, we obtain good agreements between theory and numerical results. For example, the C. Elegan neural network has the shortest relaxation time of order $\mathcal{O}(\ln N)$ in which the decay rate and MFPT agree very well in both Table 1 and Fig. 4p. For other networks, there are some deviations. The result on C. Elegan suggests that its neural network has a very efficient structure that allows a very fast transmission of signal between different neurons. This kind of insight is a valuable spin-off of our theoretical analysis.

Returning to the computation of relaxation time, we need to consider global information of the network. In general, we expect the relaxation time will decrease with increasing mean degree of the network, as shown by BA networks in Table 1. For many real world networks with high mean degree, their relaxation times are also short as they are similar to our examples of random paradigmatic networks. In contrast, for network with low mean degree, say $\langle k\rangle \approx 2$, they are better described by trees, so that the walker needs more time to move between nodes as their separation is generally larger, implying a longer relaxation time. For these networks of low mean degree, deviation from our theoretical bound is expected. The above discussions on the relation between the dynamical property in terms of relaxation time and static property in terms of mean degree can be observed in Fig. 4. Specifically, for the two similar coauthorship networks in Fig. 4 $4 \mathrm{a}$ and $4 \mathrm{~d}$, the network with smaller mean degree has larger deviation. On the other hand, both the protein interaction network and the power grid have same mean degree, but the protein interaction network has shorter relaxation time and fits better by our theory. The long relaxation time of the power grid, a consequence of its two dimensional planar nature with long diameter 22, shows that our result is not a good fit, but still provides a lower bound. For the power grid, our assumption about equilibrium states of second nearest neighbor fails as it has long relaxation time and strong local connection. Our theoretical prediction overestimates the local $\mathcal{W}(u)$ and the decay rate. The actual decay rate in this power grid is less correlated with the degree of the node, which implies that the MFPT for the high degree nodes is similar to the low degree nodes, resulting in a relatively flat distribution in Fig. 4F. For networks similar to the power grid, first passage time distribution is better described by the distance between source and destination.

Finally, we have also observed a correlation between the random walk centrality [10] and the measured decay rate. In fact, our numerical results show that the random walk centrality is approximately equal to the measured decay rate. This will be investigated further in future research.

Summary and discussion . - In conclusion, our asymptotic analysis of FPT distribution allows us to compute an accurate expression of the decay rate of FPT distribution (9) for networks with short relaxation time $\tau \ll 1 / \beta_{d}$. We have also shown that the decay rate of real world networks has similar order of magnitude as $\mathcal{P}^{\infty}\left(v_{d}\right)=k_{d} / N\langle k\rangle$ and is upper bounded by this value. This upper bound can be explained by a physical picture that local neighbors around the destination, at the quasiequilibrium state, should take the walker probability less than $\mathcal{P}^{\infty}(u)$. Moreover, we have computed the MFPT (11) for destination far from the source. A similar expression of MFPT that depends on destination degree is argued to be correct for network with $\tau \ll N$. These theoretical results on the relationship between MFPT and destination degree have been verified by numerical simulations on both paradigmatic and real world networks. Our analysis can also be readily extended to other variants of random walk problem, such as random walk in weighted networks and biased random walk 25, with short relaxation time and 
it predicts the MFPT to be

$$
\langle T(k)\rangle \gtrsim \frac{1}{\mathcal{P} \infty(k)}
$$

where $\mathcal{P}^{\infty}(k)$ is the equilibrium probability for the corresponding random walk problem.

Since $1 / \beta_{d}$ also scales linearly in $N$, the condition for networks with short relaxation time then becomes $\tau \ll N$. Assuming that this condition of short relaxation time is met, we have shown numerically that the relaxation times of many real world networks are actually short. Therefore, our analytical results are actually applicable to these real networks. We also argued that real networks with high mean degree should have short relaxation time.

Finally, we should note that although paradigmatic networks with random global links, such as the ER and BA network, show extremely short relaxation time 26 and our theory fits very well with the numerical data on these networks, we expect more complex dynamical properties from real networks, which deserve further study.

K. Y. Szeto acknowledges the support of grant CERG 602506 and 602507 . We also thank the discussion with Shi Qinwei.

References

[1] Albert R. and Barabási A. L., Rev. Mod. Phys. , 74 (2002) 47.

[2] Dorogovtsev S. N., Goltsev A. V. and Mendes J. F. F., Rev. Mod. Phys., 80 (2008) 1275.

[3] Song C., Havlin S. and Makse H. A., Nat Phys, 2 (2006) 275.

[4] Kim J. S., Goh K.-I., Salvi G., Oh E., Kahng B. and Kim D., Phys. Rev. E, 75 (2007) 016110.

[5] Frasca M., Buscarino A., Rizzo A., Fortuna L. and Boccaletti S., Phys. Rev. E , 74 (2006) 036110.

[6] Tuckwell H., Introduction to theoretical neurobiology (Cambridge University Press) 1988.

[7] Hughes B., Random walks and random environments: Volume 1: Random Walks (Oxford University Press, USA) 1995.

[8] Redner S., A guide to first-passage processes (Cambridge University Press) 2001.

[9] Condamin S., Bénichou O. and Moreau M., Phys. Rev. E, 75 (2007) 021111.

[10] Noh J. D. and Rieger H., Phys. Rev. Lett. , 92 (2004) 118701.

[11] Condamin S., Benichou O., Tejedor V., Voituriez R. and Klafter J., Nature , 450 (2007) 77.

[12] Haynes C. P. and Roberts A. P., Phys. Rev. Lett., 103 (2009) 020601.

[13] Gfeller D. and Rios P. D. L., Phys. Rev. Lett. , 100 (2008) 174104.

[14] Newman M. E. J., Strogatz S. H. and Watts D. J., Phys. Rev. E , 64 (2001) 026118.
[15] Ma C. W. and Szeto K. Y., Phys. Rev. E , 73 (2006) 047101.

[16] Baronchelli A. and Loreto V., Phys. Rev. E, 73 (2006) 026103.

[17] Newman M. E. J., Phys. Rev. E , 64 (2001) 016131.

[18] Newman M. E. J., Phys. Rev. E , 64 (2001) 016132.

[19] White J. G., Southgate E., Thomson J. N. and Brenner S., Philosophical Transactions of the Royal Society of London. Series B, Biological Sciences, 314 (1986) 1.

[20] Jeong H., Tombor B., Albert R., Oltvai Z. N. and Barabasi A.-L., Nature, 407 (2000) 651.

[21] Jeong H., Mason S. P., Barabasi A.-L. and Oltvai Z. N., Nature, 411 (2001) 41.

[22] Watts D. J. and Strogatz S. H., Nature, 393 (1998) 440.

[23] Kim D. and Kahng B., Chaos, 17 (2007) 026115.

[24] Cohen R. and Havlin S., Phys. Rev. Lett. , 90 (2003) 058701.

[25] Fronczak A. and Fronczak P., Phys. Rev. E, 80 (2009) 016107.

[26] Samukhin A. N., Dorogovtsev S. N. and Mendes J. F. F., Phys. Rev. E, $7 \mathbf{7}$ (2008) 036115. 